\title{
Riscos psicossociais no trabalho de auxiliares de cozinha de uma indústria
}

\author{
Riesgos psicosociales en el trabajo de los auxiliares de cocina en una industria
}

Psychosocial risks in the work of kitchen assistants of an industry

Fabieli Tais Rüiedell
$\begin{aligned} & \text { Faculdade Meridional (IMED), Passo Fundo-RS/Brasil } \\ & \text { ORCID: 0000-0002-2251-3531 } \\ & \text { E-mail: fabieliruedell8@gmail.com }\end{aligned}$
$\begin{aligned} & \text { Faculdade Meridional (IMED), Passo Fundo- RS/Brasil } \\ & \text { ORCID: 0000-0002-2804-1045 }\end{aligned}$
E-mail: julia.goncalves@imed.edu.br

\begin{abstract}
Resumo
A Psicodinâmica do Trabalho, abordagem teórica deste estudo, destaca a importância e influência do contexto de trabalho nos processos de saúde e adoecimento do trabalhador. Nessa perspectiva, os riscos psicossociais no trabalho são compreendidos como fatores que se relacionam com as percepções dos trabalhadores acerca da organização do trabalho, da gestão, do sofrimento e dos problemas emocionais, físicos e psíquicos. Esta pesquisa mapeou os riscos psicossociais em auxiliares de cozinha de uma indústria localizada no interior Rio Grande do Sul. Foi realizada uma pesquisa quantitativa e descritiva, em que 28 auxiliares de cozinha responderam à um questionário de dados sociodemográficos e ao Protocolo de Avaliação dos Riscos Psicossociais no Trabalho (PROART). Os dados foram analisados a partir da estatística descritiva. Os resultados obtidos destacaram os elevados riscos psicossociais presentes na organização do trabalho, em especial relacionados as condições, prazos, ritmos, normas, responsabilidades e divisão do trabalho. Por outro lado, o estilo de gestão coletivista foi assinalado como o mais presente, ou seja, há uma percepção de que os gestores da empresa valorizam o trabalho em grupo, reconhecem as conquistas como mérito de todos e levam em conta o bem-estar dos trabalhadores. Estudos com categorias profissionais que, muitas vezes, são invisibilizadas - com a de auxiliares de cozinha - são essenciais, pois propiciam um reconhecimento da importância da função para o funcionamento da empresa, bem como podem fornecer subsídios teóricos para que a gestão promova ações de melhoria das condições e da organização do trabalho desses profissionais.
\end{abstract}

Palavras-chaves: Trabalho; Fatores de riscos; Saúde do trabalhador.

\section{Resumen}

La Psicodinámica del Trabajo, abordaje teórico de este estudio, destaca la importancia e influencia del contexto laboral en los procesos de salud y enfermedad del trabajador. Desde esta perspectiva, los riesgos psicosociales en el trabajo son entendidos como factores que se relacionan con las percepciones de los trabajadores sobre la organización, la gestión, el sufrimiento y los problemas emocionales, físicos y psicológicos del trabajo. Esta investigación mapeó los riesgos psicosociales en los ayudantes de cocina en una industria del interior de Rio Grande do Sul. Se realizó una investigación cuantitativa y descriptiva, en la que 28 ayudantes de cocina respondieron un cuestionario de datos sociodemográficos y el Protocolo de Evaluación de Riesgo Psicosocial en el Trabajo (PROART). Los datos se analizaron mediante estadística descriptiva. Los resultados obtenidos destacaron los altos riesgos psicosociales presentes en la organización del trabajo, especialmente relacionados con las condiciones, plazos, ritmos, reglas, responsabilidades y división del trabajo. Por otro lado, el estilo de gestión colectivista fue identificado como el más presente, o sea, los directivos de la empresa valoran el trabajo en grupo, reconocen los logros como un mérito de todos y tienen en cuenta el bienestar de los trabajadores. Los estudios con categorías profesionales muchas veces invisibles - como de ayudantes de cocina- son fundamentales porque traen un reconocimiento de la importancia de la función para el funcionamiento de la empresa, así como pueden proveer subsidios teóricos para que la gerencia promueva acciones para mejorar las condiciones y organización del trabajo de estos profesionales. 
Palabras clave: Trabajo; Factores de riesgo; Salud laboral.

\begin{abstract}
The Psychodynamic of Work, the theoretical approach of this study, highlights the importance and influence of the work context in the worker's health and illness. From this perspective, psychosocial risks at work are understood as factors that relate to workers' perceptions about work organization, management, suffering and emotional, physical and psychological problems. This research mapped the psychosocial risks in kitchen assistants in an industry located in the interior of Rio Grande do Sul. A quantitative and descriptive research was conducted, in which 28 kitchen assistants answered a questionnaire of sociodemographic data and the Psychosocial Risk Assessment Protocol in the Work (PROART). The data were analyzed using descriptive statistics. The results
\end{abstract}

obtained highlighted the high psychosocial risks present in the organization of work, especially related to conditions, deadlines, rhythms, rules, responsibilities and division of labor. On the other hand, the collectivist management style was identified as the most present, that is, there is a perception that the company's managers value group work, recognize the achievements as a merit of all and take into account the well-being of workers. Studies with professional categories that are often invisible - with the help of kitchen assistants - are essential because they provide recognition of the importance of the function for the functioning of the company, as well as they can provide theoretical subsidies so that management promotes actions to improve conditions and work organization of these professionals.

Keywords: Work; Risk factors; Occupational health.

\section{Introdução}

O trabalho é fonte de identidade e o principal meio de socialização, onde indivíduos constroem suas experiências e relacionamentos (Tolfo, 2019) e se percebem como integrantes e pertencentes à um contexto maior. As mudanças sociais, econômicas, políticas e tecnológicas que ocorreram e ainda ocorrem no mundo do trabalho, tornam o trabalho ainda mais central, pois somos incapazes de ficarmos sem ele, tanto por uma questão de sobrevivência e subsistência quanto pelos sentidos e significados que lhe são atribuídos (Jacinto, Huck, Silva \& Tolfo, 2020; Ruiz \& Araújo, 2012; Tolfo, 2019).

Ao analisar o contexto nacional, identifica-se que os trabalhos (e empregos) no Brasil possuem um foco na produtividade, o que indica um excesso de horas de trabalho, excessiva pressão e expectativas irrealizáveis (Álvarez, 2012; Conte, 2003; Gomez \& Costa, 1997; Jacinto et al., 2020; Zanelli \& Kanan, 2018) características essas que podem ser geradoras de sintomas emocionais, físicos e fisiológicos na saúde dos trabalhadores (Gomez \& Costa, 1997; Zanelli \& Kanan, 2018). Além disso, há exigências de que o trabalhador seja polivalente e capaz de realizar, cada vez mais, atividades complexas e diversificadas (Jacinto et al., 2020). Esses fatores evidenciam que o cuidado com os aspectos presentes na relação entre a saúde e o trabalho em muitas organizações não é central (Tolfo, 2019). Entretanto, a atenção a saúde do trabalhador é a melhor maneira de impedir acidentes e controlar riscos, além disso, o incentivo ao trabalho em equipe, aflora a ideia de que existe ajuda mútua no enfrentamento de situações de risco (Gomez, Vasconcellos, \& Machado, 2018) e traz repercussões positivas para o ambiente de trabalho.

As novas características do mundo do trabalho e suas consequências no desempenho e saúde dos trabalhadores fomentaram a discussão sobre uma nova categoria de aspectos envolvidos na saúde e segurança laboral (Álvarez, 2012; Gomez \& Costa, 1997; Jacinto et al., 2020; Ruiz \& Araújo, 2012). Os fatores psicossociais são compreendidos como elementos da estrutura física e psicológica laboral e que são determinados pela gestão e organização do trabalho (Carlotto et al., 2018; Jacinto et al., 2020). Os fatores psicossociais pressupõem interações entre aspectos individuais (tais como a capacidade, as necessidades e percepções do trabalhador) e sociais (por exemplo o ambiente de trabalho, as condições organizações, a cultura e as condições de trabalho) (Zanelli \& Kanan, 2018) e podem ser categorizados como de risco ou protetivos ao trabalhador (Carlotto et al., 2018; Jacinto et al., 2020). Os fatores favoráveis ou protetivos são os que promovem o bem-estar e 
a produtividade, enquanto os de risco são os que podem causar algum prejuízo ou dano ao trabalhador, afetando sua saúde e, principalmente, seu psicológico (Álvarez, 2012; Jacinto et al., 2020).

A Organização Internacional do Trabalho (OIT) define os riscos psicossociais como interações e condições de trabalho que, quando em contato com a subjetividade do trabalhador, podem causar danos à saúde física e/ ou psíquica (Carlotto et al., 2018; Jacinto et al., 2020; Ruiz \& Araújo, 2012). Estima-se que as maiores repercussões são na saúde mental, destacando a ansiedade, a depressão, o estresse no trabalho (Duarte, 2018), o desenvolvimento de adições e até o suicídio (Zanelli, \& Kanan, 2018). Cabe destacar que, os riscos psicossociais não derivam apenas da percepção do trabalhador e podem ser tão visíveis que acabam desaparecendo da própria percepção, pois são entendidos como inerentes ao trabalho (OIT, 2010). Os riscos psicossociais do trabalho envolvem diversos aspectos, tais como a sobrecarga quantitativa, a carga qualificada insuficiente, a falta de controle sobre $\mathrm{o}$ trabalho, a falta de apoio social em casa ou por parte da chefia e colegas de trabalho, os estressores físicos, os papéis conflitantes, dentre outros (Álvarez, 2012; Duarte, 2018). Dessa forma, são exemplos de riscos psicossociais a pressão, o estresse, o cansaço físico e mental e os conflitos interpessoais, condições que são capazes de transformar a rotina de trabalhadores acarretando em prejuízo a sua saúde (Carlotto et al., 2018; Jacinto et al., 2020; Pucci, Kanan, \& Silva, 2017). Segundo Zanelli e Kanan (2018) é fundamental que os riscos sejam reconhecidos, monitorados e prevenidos para que ocorra a promoção de saúde, bem-estar e qualidade de vida dos trabalhadores e das organizações.

Dentre as abordagens teóricas quem detém-se aos estudos em saúde do trabalhador (Jacinto et al., 2020), optou-se por utilizar a Psicodinâmica do Trabalho (PDT). Esta perspectiva acredita que é possível um equilíbrio e saúde para o trabalhador mesmo em condições de trabalho desagradáveis. A PDT busca uma análise crítica do trabalho, buscando reorganizá-lo e atuando, prioritariamente, de forma coletiva (Merlo \& Mendes, 2009). A PDT compreende os riscos psicossociais a partir de um conjunto de fatores presentes na organização do trabalho, nos estilos de gestão, no sofrimento relacionado ao trabalho e nos danos - físicos, psíquicos e sociais, que impactam em adoecimento mental pelo trabalho (Facas \& Mendes, 2018).

Os riscos psicossociais estão presentes no trabalho de diferentes ocupações e categorias profissionais, um exemplo é em auxiliares de cozinha, em que um olhar atento pode evitar alguns dos problemas de saúde causados pelo ambiente. Em pesquisa com auxiliares de cozinha da cidade de Natal/RN, Mendes (2016) comprovou que o ramo da alimentação coletiva vem crescendo a cada dia. Apesar disso, há um descaso com estes trabalhadores, submetidos a demissões recorrentes, contratos temporários, salários baixos, baixa autoestima e uma insegurança laboral permanente. Já o estudo de Rodrigues e Santana (2010) com auxiliares de cozinha de uma cidade do interior da Bahia evidenciou que a qualidade nos produtos e dos resultados do trabalho destes profissionais também está relacionada a segurança, assim são estabelecidas regras de higiene, boas práticas de fabricação e a utilização de equipamento de proteção individual para garantir a qualidade dos alimentos. Esse estudo demonstrou que nem sempre a segurança dos trabalhadores gera conforto, as vezes uma luva pode incomodar a manipulação dos materiais e alimentos de seu serviço, mas é necessária sua utilização. Além disso, resultados evidenciaram que o desempenho das atividades tende a trazer desgastes emocionais, pela pressão de entregas com horários, e a falta de valorização.

Frente a esse cenário, o objetivo deste estudo foi mapear os riscos psicossociais em auxiliares de cozinha de uma indústria em uma cidade do interior do estado do Rio Grande do Sul. O interesse em investigar a temática deuse pela experiência profissional da primeira autora com esses profissionais. Além disso, poucas pesquisas dedicam-se a estudar a categoria, que muitas vezes ocupa um lugar de 
invisibilidade dentro das organizações, mas as mesmas possuem importante função sendo as responsáveis pelas preparações, acabamentos e apresentação dos pratos, bem como a manutenção do local de trabalho organizado e limpo para os demais trabalhadores.

\section{Método \\ Participantes}

O presente estudo trata-se de uma pesquisa quantitativa e descritiva de levantamento. O cenário do estudo é uma empresa especializada em projetos de alimentação para o mercado corporativo com matriz no Paraná. Essa empresa atua há 24 anos e, atualmente possui filiais nos estados do Rio Grande do Sul, Santa Catarina, Paraná, São Paulo, Rio de Janeiro e Espírito Santo. Ao total, conta com 460 funcionários nas funções de departamento pessoal, administrativos, financeiros, compras, vendas, e restaurantes, com chefes de cozinha, cozinheiras, auxiliares de cozinha, auxiliares de higienização e líderes de operações. Em específico, esta pesquisa foi realizada em uma filial que realiza suas atividades em uma cidade do interior do estado do Rio Grande do Sul e conta com quarenta trabalhadores, sendo destes, trinta auxiliares de cozinha.

Como critérios de inclusão foram estabelecidos: ser maior de 18 anos; atuar como auxiliar de cozinha por no mínimo seis meses; estar, no momento da coleta dos dados, trabalhando na empresa pesquisada; e aceitar participar de forma espontânea e voluntária do estudo. Já o único critério de exclusão adotado foi, durante o período de coleta, estar em gozo de férias ou licença de qualquer motivo. Dos trinta auxiliares de cozinha, um trabalhador estava em período de férias e outro se recusou a participar da pesquisa. Dessa forma, participaram 28 auxiliares de cozinha.

A partir da caracterização da amostra identificou-se que mais da metade das participantes possuem baixa escolaridade, sendo que $67,9 \%$ cursaram apenas o ensino fundamental (completo ou incompleto), $57,10 \%$ são casados ou estão em união estável e $96,4 \%$ estavam em contratos de trabalho efetivos. Destaca-se que $100 \%$ das participantes eram do sexo feminino, a idade média foi de 41,7 anos $(D P=10,55$; $\operatorname{Min}=22$; Máx $=62)$ e o tempo de serviço na empresa era de, em média, 17,9 meses $(D P=9,41 ; \operatorname{Min}=6$; Máx = 34).

\section{Instrumentos}

Para a coleta de dados foi utilizado o questionário de dados sociodemográficos com questões relacionadas ao nível de instrução, estado civil, tempo de serviço e o tipo de contrato de trabalho, com ele buscou-se caracterizar os participantes da pesquisa. Após foi aplicado Protocolo de Avaliação dos Riscos Psicossociais no Trabalho - PROART (Facas \& Mendes, 2018) - com autorização prévia do primeiro autor. $\mathrm{O}$ instrumento, construído e validado no Brasil, mapeia os riscos psicossociais no trabalho, possuindo como objetivos específicos: investigar as características da organização do trabalho, avaliar o estilo de gestão da organização, levantar indicadores de sofrimento patogênico no trabalho e identificar os danos físicos e psicossociais decorrentes do trabalho (Facas, 2013; Facas \& Mendes, 2018).

A primeira versão PROART foi construída com 95 itens (Facas, 2013). Após processos de validação, a estrutura do protocolo sofreu pequenos ajustes, tendo sua versão final com 91 itens distribuídos em quatro escalas: (1) Escala da Organização do Trabalho - EOT (19 itens); (2) Escala de Estilos de Gestão - EEG (21 itens); (3) Escala de Indicadores de Sofrimento no Trabalho - EIST (28 itens) e (4) Escala de Danos Relacionados ao Trabalho EDT (23 itens). As opções de resposta são em escala tipo Likert de 5 pontos (de $1=$ nunca a 5 = sempre).

As escalas são divididas em fatores e foram analisadas a partir dos resultados das médias, conforme a indicação dos próprios autores do instrumento. A EOT com dois fatores: (1) divisão das tarefas e (2) divisão social do trabalho possui como indicadores para avaliação de média: risco alto de 1 a 2,29; 
risco médio de 2,3 a 3,69 e risco baixo de 3,7 a 5 - destaca-se que a avaliação desta escala é invertida as demais, quando maior média, menor risco. A EEG também é dividida em dois fatores: (1) gestão individualista e (2) gestão coletivista, em que os valores da média de respostas para se concluir sobre a presença de cada estilo são de 1 a 2,5 pouco característico; de 2,5 a 3,5 presença moderada e de 3,5 a 5 padrão predominante. A EIST é dividida em três fatores: (1) falta de sentido do trabalho; (2) esgotamento mental; e (3) falta de reconhecimento; assim como EDT em que os três fatores são: (1) danos psicológicos; (2) danos sociais; e (3) danos físicos. Estas duas escalas são analisadas com as seguintes medidas: risco baixo de 1 a 2,29; risco médio de 2,30 a 3,69 e; risco alto de 3,7 a 5 (Facas, 2013; Facas \& Mendes, 2018).

\section{Procedimentos Éticos e de Coleta de Dados}

O contato inicial foi realizado com a Direção geral da empresa, apresentando a proposta de pesquisa e solicitando um Termo de consentimento institucional. Após a autorização, o projeto foi submetido à apreciação pelo Comitê de Ética em Pesquisa da IMED recebendo número de aprovação 3.460.431. A pesquisa respeitou aos preceitos éticos das resoluções do Conselho Nacional de Saúde nº 466/2012 e nº 510/2016.

Os participantes foram convidados a participarem durante seu horário de trabalho, conforme acordado com a supervisão da filial em que a pesquisa ocorreu. No momento do convite foi explicado todos os detalhes: como a pesquisa seria realizada, quais riscos e benefícios envolvidos, bem como os direitos e demais questões éticas pertinentes. Os trabalhadores que aceitaram participar da pesquisa preencheram e assinaram o Termo de Consentimento Livre e Esclarecido.

Os trabalhadores foram liberados, pela gestão da empresa, para responderem aos instrumentos de dois em dois, embora cada um preenchia os instrumentos de forma individual e em mesa separada. No momento da aplicação foi lido com os participantes o cabeçalho do
PROART e explicado a forma de respondê-lo. Foi informado que não existiam respostas certas ou erradas e o questionário em si não seria apresentado para a empresa, apenas o relatório final sem a identificação dos participantes. A aplicação demorou em média trinta minutos para cada dupla. Ao final os instrumentos eram guardados em um envelope escuro, sendo misturado com os demais, garantindo o anonimato do participante.

\section{Análise de dados}

Os dados foram primeiramente digitados no Excel e posteriormente analisados e computados no programa Statistical Package for the Social Sciences (SSPS 23.0). A análise descritiva dos instrumentos objetivou apresentar e descrever os aspectos relevantes no conjunto de dados. Assim, abarcou medidas de tendência central (média, desvio padrão e frequência).

\section{Resultados e Discussão}

A denominação, fatores psicossociais relacionados ao trabalho, surgiu sob a perspectiva de análise dos fenômenos ocupacionais, apesar disso envolve variáveis externas ao ambiente de trabalho (Zanelli \& Kannan, 2018), tais como as percepções e experiências dos trabalhadores e são influenciados tanto pelo contexto laboral quanto pelo cenário social e econômico (Jacinto et al., 2020). Identificar os aspectos objetivos que envolvem, em especial, os riscos psicossociais presentes no ambiente laboral permite construir estratégias para diminuição do estresse, das doenças físicas e mentais relacionadas ao trabalho e dos problemas relativos ao desempenho (Carlotto et al., 2018). Para Zanelli e Kanan (2018) pode-se compreender como risco tudo aquilo com potencial para gerar dano ao indivíduo e sua saúde. Dessa forma, as análises descritivas provindas de um instrumento de autorrelato como o PROART (Facas \& Mendes, 2018) permitem mapear os riscos psicossociais presentes no trabalho desses auxiliares de cozinha. A Tabela 1 apresenta os resultados 
gerais das quatro escalas que compõem o

protocolo aplicado.

Tabela 1

Estatística descritiva referente as escalas que compõem o PROART

\begin{tabular}{llll}
\hline Escala & Média & DP & Análise \\
\hline Escala de Indicadores de Sofrimento no Trabalho (EIST) & 2,03 & 0,88 & Risco Baixo \\
Escala de Danos Físicos e Psicossociais no Trabalho (EDT) & 2,19 & 0,85 & Risco Baixo \\
Escala da Organização do Trabalho (EOT) & 3,24 & 0,62 & Risco Médio \\
Escala de Estilos de Gestão (EEG) & 3,29 & 0,33 & Presença Moderada \\
\hline
\end{tabular}

Nota. $\mathrm{N}=28 ; \mathrm{DP}$ = Desvio Padrão.

A Escala de Estilos de Gestão (EEG) avalia as formas de agir, sentir e pensar dentro da empresa, ou seja, identifica o quanto o estilo de gestão adotado pela organização busca que os trabalhadores entreguem o melhor de si e o quanto valoriza o trabalho em equipe (Facas, 2013). Já a Escala da Organização do Trabalho (EOT) se refere as condições de trabalho, prazos, ritmos e normas (Facas, 2013) e demonstrou que a média de seus itens é classificada em risco médio. Os valores indicaram que as respostas da EEG e EOT se concentraram nas alternativas às vezes $\mathrm{e}$ frequentemente (3 e 4, respectivamente), representando a necessidade de estar alerta aos riscos psicossociais identificados por estas escalas.

A Escala de Indicadores de Sofrimento no Trabalho (EIST) que avalia as vivências em relação ao trabalho atual indicando o sentido do trabalho e o reconhecimento percebido pelos trabalhadores por parte da empresa (Facas, 2013), apresentou a menor média. Da mesma forma que a Escala de Danos Físicos e Psicossociais no Trabalho (EDT) que avalia os problemas físicos, psicológicos e sociais (Facas, 2013). As respostas destas duas escalas (EIST e EDT) se concentraram nas alternativas raramente e as vezes ( 2 e 3 respectivamente), indicando baixos riscos psicossociais relacionados aos aspectos descritos e avaliados por elas.

Os baixos valores dos desvios-padrão em todas as escalas - entre 0,33 e 0,88 -, indicam homogeneidade nas respostas entre as participantes. Segundo Facas (2013) quando o desvio-padrão é menor ou igual a 1,00 é porque não houve grande dispersão nas respostas, ou seja, todos que responderam lançaram valores próximos ao da média apresentada.

\section{A Organização do Trabalho}

Os fatores relacionados à organização do trabalho dizem respeito à satisfação de necessidades e expectativas dos trabalhadores, como as condições de trabalho, as tarefas a serem desenvolvidas, a estrutura física e material do local e mesmo as relações interpessoais estabelecidas (Jacinto et al., 2020; Ruiz \& Araújo, 2012). Para a PDT é na organização do trabalho que as ações e intervenções de promoção de saúde e mitigação de sofrimento devem ser realizadas, já que a abordagem visa, prioritariamente, ações para o coletivo de trabalho e não aos indivíduos isoladamente (Merlo \& Mendes, 2009). Para Vieira, Mendes e Merlo (2013) a PDT considera a organização do trabalho como uma divisão de trabalho, sendo homem e o mundo do trabalho divididos, tal divisão é para organizar o homem por meio das vivências do dia a dia de prazer e sofrimento.

Os itens da EOT investigaram a compreensão dos trabalhadores sobre suas tarefas, as condições materiais da organização, os prazos estabelecidos, os ritmos, as normas e a autonomia no trabalho (Facas, 2013; Facas, Duarte, Mendes, \& Araújo, 2015). Os dois fatores que compõem a escala - (1) divisão das tarefas e (2) divisão social do trabalho - seguem a definição de organização do trabalho proposta pela PDT, em que todo trabalho é dividido em 
prescrito (que antecede as tarefas e está descrito e previsto) e o trabalho real (que é o que o trabalhador realmente faz, as atividades que ele desenvolve) (Vieira et al., 2013). Dessa forma, o fator divisão das tarefas avalia os prazos e as condições oferecidas para a execução das tarefas, já o fator divisão social do trabalho avalia a comunicação e a autonomia dentro da empresa (Facas \& Mendes, 2018).

Os itens desta escala foram analisados seguindo os valores para avaliação de média indicados pelos autores do instrumento, sendo eles: a) entre 1,00 a 2,29 indica um resultado negativo e a presença de altos riscos psicossociais, o que infere a necessidade de intervenções imediatas; b) entre 2,30 e 3,69representa um resultado mediano, ou seja, um estado de alerta (situação limite) para os riscos psicossociais no trabalho; e c) entre 3,70 e 5,00 - indica um resultado positivo, representa baixos riscos psicossociais, ou seja, são aspectos a serem mantidos e potencializados na organização do trabalho (Facas \& Mendes, 2018). A Tabela 2 destaca os resultados mais representativos da escala.

Tabela 2

Escala da organização do trabalho (EOT)

\begin{tabular}{llll}
\hline Fator - Item & Média & DP & Risco \\
\hline Divisão de Tarefas & $\mathbf{2 , 9 0}$ & $\mathbf{0 , 8 1}$ & Médio \\
\hline O número de trabalhadores é suficiente para a execução das tarefas. & 1,96 & 1,35 & Alto \\
O ritmo de trabalho é adequado. & 2,25 & 1,35 & Alto \\
\hline Divisão Social do trabalho & $\mathbf{3 , 4 4}$ & $\mathbf{0 , 6 3}$ & Baixo \\
\hline Os funcionários participam das decisões sobre o trabalho. & 2,04 & 0,96 & Alto \\
\hline
\end{tabular}

Nota. $\mathrm{N}=28 ; \mathrm{DP}=$ Desvio Padrão

O fator divisão social do trabalhou apresentou média mais elevada $(M=3,44 ; D P$ $=0,63)$. Resultado semelhante foi encontrado na pesquisa realizada por Pacheco e Silva (2018) com os servidores de uma universidade pública do norte do país que objetivou compreender o modo de organização do trabalho e seus impactos nos trabalhadores. Nesse fator, o item sobre a participação dos trabalhadores nas decisões sobre o trabalho mostrou-se como alto risco psicossocial. A participação nas decisões que afetam o próprio trabalho é um potencial fator protetivo, pois quando o trabalhador pode expressar sua opinião e auxiliar nas tomadas de decisão ele sente-se reconhecido e engajado, o que traz efeitos em sua qualidade de vida e, consequentemente, na produtividade (Conte, 2003; Jacinto et al., 2020).

No fator divisão de tarefas, os itens que descreviam sobre a quantidade de trabalhadores para a execução das tarefas e a adequação do ritmo de trabalho apresentaram-se como alto risco psicossocial. O aumento no volume e ritmo das atividades laborais representam um risco de intensificação do trabalho que pode repercutir em quadros de adoecimento
(Álvarez, 2012; Jacinto et al., 2020). Os itens presentes na EOT permitem conhecer o trabalho, como ele é realizado e quais relações sociais o envolvem, ou seja, os diferentes elementos presentes na organização do trabalho, e essa identificação é essencial para o combate aos danos à saúde (Ruiz \& Araújo, 2012), diminuição dos prejuízos financeiros, das vivências de desprazer e de desagregação social (Zanelli \& Kanan, 2018).

\section{Os Estilos de Gestão}

Certos estilos e perspectivas de gestão, que enfatizam determinadas características do ambiente laboral, possuem potencial próprio para causar danos à saúde (Carlotto et al., 2018). A EEG identifica o modelo de trabalho e as percepções compartilhadas na organização, entendendo-as como parte constituinte do estilo de gestão da organização (Facas, 2013). É importante destacar que deficiências da gestão, estilo de comando e controle e contexto de trabalho precário são fatores de risco importantes em um ambiente ocupacional (Jacinto et al., 2020). A EEG identifica o estilo de gestão - coletivista ou individualista predominante no modo como o trabalho está 
organizado (Pacheco \& Silva, 2018). Por se tratar de uma escala de cinco pontos, considerou-se que valores próximos ao ponto médio da escala $(3,00)$ significam uma presença moderada de determinado estilo, enquanto que, acima de 3,50 o padrão é predominante e abaixo de 2,50, pouco característico (Facas \& Mendes, 2018). A Tabela 3 destaca os resultados mais representativos da escala.

Tabela 3

Escala de Estilos de Gestão (EEG)

\begin{tabular}{llll}
\hline Fator - Item & Média & DP & Presença do Estilo \\
\hline Gestão Individualista & $\mathbf{2 , 9 3}$ & $\mathbf{0 , 7 4}$ & Presença Moderada \\
\hline A hierarquia é valorizada nesta organização. & 3,61 & 1,42 & Predominante \\
O ambiente de trabalho se desorganiza com mudanças. & 3,70 & 0,93 & Predominante \\
É creditada grande importância para as regras nesta organização. & 3,71 & 1,21 & Predominante \\
Há forte controle no trabalho. & 3,86 & 1,11 & Predominante \\
\hline Gestão Coletivista & $\mathbf{3 , 3 6}$ & $\mathbf{0 , 5 3}$ & Presença Moderada \\
\hline Somos incentivados pelos gestores a buscar novos desafios. & 3,54 & 1,20 & Predominante \\
Para esta organização, o resultado do trabalho é visto como uma realização & 3,96 & 1,13 & Predominante \\
do grupo. & 3,96 & 1,42 & Predominante \\
Os gestores se preocupam com o bem-estar dos trabalhadores. & 4,00 & 1,05 & Predominante \\
A inovação é valorizada nesta organização. & 4,43 & 1,28 & Predominante \\
O trabalho coletivo é valorizado pelos gestores. & 4,54 & 0,99 & Predominante \\
O mérito das conquistas na empresa é de todos. & & &
\end{tabular}

Nota. $\mathrm{N}=28 ; \mathrm{DP}=$ Desvio Padrão

Para os dois tipos de gestão avaliados identificou-se presença moderada das características descritas nos itens do protocolo, apesar disto, a "gestão coletivista" obteve a média mais alta $(M=3,36 ; D P=0,53)$. $\mathrm{O}$ fator "gestão individualista" apresenta itens que revelam que as decisões se concentram nas mãos dos gestores, que a organização valoriza muito o cumprimento de regras e que existe um sistema rígido e de forte controle no trabalho. Este estilo é caracterizado por pessoas que não sabem reconhecer o trabalho do outro, somente o seu (Facas \& Mendes, 2018). Nesse fator, as afirmações que obtiveram maior média, indicando serem predominantes na organização pesquisada, referiam-se à valorização da hierarquia, ao ambiente pouco adaptável a mudanças, a importância creditada as regras e ao controle no trabalho. Uma gestão individualista é a realidade de muitos lugares em que $o$ gestor possui dificuldade de reconhecimento e valorização do trabalho de sua equipe (Pacheco \& Silva, 2018).

Já no fator "gestão coletivista" os itens se referem ao trabalhar em equipe e o investimento da gestão para que isto ocorra, também retrata o quanto a gestão favorece o bem-estar dos trabalhadores, tem flexibilidade nas normas e procura prover o reconhecimento de todos. Neste estilo de gestão o mérito e o esforço do trabalho são do grupo (Facas \& Mendes, 2018). Esse fator obteve mais itens classificados como predominantes, estes se referiam ao incentivo a busca por novos desafios e a inovação, o reconhecimento do trabalho em grupo e do coletivo, a preocupação com o bem-estar dos trabalhadores e a divisão do mérito das conquistas entre todos da empresa. Conforme Amorim (2010) os trabalhadores tendem a desenvolver seu trabalho melhor se forem tratados de maneira justa pela empresa, valorizando o sucesso do trabalho. Com isso, o trabalho pode ser fonte de prazer desde que se mantenha foco no bemestar dos trabalhadores (Vieira et al., 2013). Organizações em que a percepção dos trabalhadores reflete um ambiente de apoio e ajuda, com flexibilidade e tolerância nas relações interpessoais e práticas de gestão são potencialmente mais saudáveis (Jacinto et al., 2020). Além disso, as vivências de prazer no trabalho são observadas quando a organização do trabalho é flexível e os trabalhadores sentem-se úteis, participativos, produtivos e valorizados (Merlo \& Mendes, 2009). 
A gestão coletivista estabelece relações de igualdade entre gestores e funcionários, assim o trabalhador é valorizado pelo seu trabalho (Faria, 2017). A partir disto, a gestão coletivista pode ser superior que a individualista, pois propicia o bem-estar dos trabalhadores e o trabalho em equipe, em que todos são incluídos na organização. Além disso, ter uma gestão que incentiva o trabalho em grupo e que se possa contar com o colega frente a situações desafiadoras beneficia na diminuição de riscos e acidentes de trabalho (Gomez, Vasconcellos, \& Machado, 2018). É essencial a participação efetiva dos gestores, sua qualificação e atuação comprometida com uma cultura organizacional que incentive e promova ações adequadas e eficazes a fim de conter os riscos psicossociais laborais (Zanelli \& Kanan, 2018). Conforme Ruiz e Araújo (2012) o reconhecimento e a ampliação da capacidade de análise e gestão coletiva sobre o próprio trabalho é o que permitirá avançarmos na direção de outras possibilidades de abordagem dos riscos psicossociais, para uma dimensão mais ampla e completa.

\section{Indicadores de Sofrimento no Trabalho}

O sofrimento no trabalho pode estar relacionado a diferentes necessidades, sendo específico de pessoa para pessoa (Silva \& Garcia, 2018) apesar disto, o olhar para o contexto de trabalho como um todo é fundamental para reconhecer os possíveis fatores desencadeadores deste sofrimento (Merlo \& Mendes, 2009). As condições oferecidas pela organização do trabalho e pela gestão dos aspectos técnicos e burocráticos são determinantes para o manejo e o destino do sofrimento mental do trabalhador frente aos riscos psicossociais no ambiente de trabalho (Jacinto et al., 2020).

Em especial, o trabalho, as normas e as leis da organização podem causar um desequilíbrio mental dependendo do trabalhador. Os itens da EIST representam o modo que as pessoas sentem, pensam e trabalham na empresa. As afirmações da escala referem-se ao sofrimento dos trabalhadores, as vivências de desqualificação, inutilidade e sofrimento psíquico (Facas, 2013). A avaliação dos valores da média considerou que resultados entre 3,70 e 5,00 indicavam um resultado negativo, evidenciando altos riscos psicossociais e a demanda por ações imediatas; entre 2,30 e 3,69 apresentam um resultado mediano, é um estado de alerta/situação limite para os riscos psicossociais no trabalho; e entre 1,00 a 2,29 representam um resultado positivo, ou seja, baixos riscos psicossociais (Facas \& Mendes, 2018). A Tabela 4 destaca os resultados mais representativos da escala.

Tabela 4

Escala de Indicadores de Sofrimento no trabalho (EIST)

\begin{tabular}{llll}
\hline Fator - Item & Média & DP & Risco \\
\hline Falta de Sentido do Trabalho & $\mathbf{1 , 6 8}$ & $\mathbf{0 , 8 6}$ & Baixo \\
\hline Esgotamento Mental & $\mathbf{2 , 2 5}$ & $\mathbf{1 , 0 3}$ & Baixo \\
\hline Meu trabalho me desanima & 2,37 & 1,36 & Médio \\
Meu trabalho me sobrecarrega & 2,61 & 1,34 & Médio \\
Meu trabalho é desgastante & 2,89 & 1,47 & Médio \\
Meu trabalho é cansativo & 3,18 & 1,44 & Médio \\
\hline Falta de Reconhecimento & $\mathbf{1 , 8 5}$ & $\mathbf{0 , 7 6}$ & Baixo \\
\hline Falta-me liberdade para dizer o que penso sobre meu trabalho & 2,37 & 1,47 & Médio \\
Meus colegas desvalorizam meu trabalho & 2,39 & 1,37 & Médio \\
\hline
\end{tabular}

$\mathrm{N}=28 ; \mathrm{DP}=$ Desvio Padrão

Os três fatores que compõem a escala são: a falta de sentido do trabalho $(M=1,68 D P$ $=0,86)$ que refere-se a sentimentos de inutilidade, o trabalho que não tem importância e sentido para si mesmo ou para a sociedade; o esgotamento mental $(M=2,25 D P=1,03)$ que se caracteriza por sentimentos de desânimo e injustiça em seu trabalho; e a falta de 
reconhecimento $(M=1,85 ; D P=0,76)$ que é descrito por itens que revelam desvalorização dos trabalhadores pelos supervisores e a falta de liberdade para falar sua opinião (Facas \& Mendes, 2018).

No fator "falta de sentido no trabalho" não houveram itens indicados como risco psicossocial médio ou alto. Esse aspecto é positivo pois indica que os respondentes do protocolo identificam sentido em seu trabalho. Para Tolfo (2019) o sentido do trabalho é considerado um fenômeno polissêmico e reflete a relação entre o indivíduo e as atividades das quais ele se apropria em seu trabalho. Esse fenômeno para a PDT é reconhecido como uma representação individual e/ou coletiva, onde o processo de produção do trabalho é apreciado pelas vivências do ambiente (Vieira et al., 2013). No fator "esgotamento mental" também não foram identificadas afirmações consideradas como alto risco psicossocial. Já os itens que referiam ao trabalho ser fonte de desânimo, sobrecarrega, desgaste e cansaço indicaram risco médio. Estes aspectos podem ser compreendidos como consequência da intensificação do trabalho (Jacinto et al., 2020) identificada na organização do trabalho desses auxiliares de cozinha. Conforme Ceribeli e Rocha (2019), o esgotamento mental pode estar relacionado a não realização profissional e pessoal, o que resulta em sentimentos negativos e dores como o próprio desgaste e sobrecarga identificados como risco na escala utilizada.

No fator "falta de reconhecimento" dois itens foram identificados com risco médio: a falta de liberdade para dizer o que se pensa sobre o trabalho e sobre os colegas desvalorizam seu trabalho. O reconhecimento no trabalho é fundamental para o enfrentamento dos fatores de risco, já que com ele o investimento pessoal adquire um sentido especial para aquele trabalhador (Jacinto et al., 2020). O reconhecimento para PDT é um elemento importante para a saúde, pois para o trabalhador estar bem consigo mesmo e feliz ele precisa ter motivação, satisfação e ser reconhecido pelo seu trabalho, assim conseguirá fazer tudo bem feito (Lino, 2018).

\section{Escala de Danos Relacionados ao Trabalho}

As repercussões de um contexto de trabalho inadequado, tais como transtornos mentais e lesões físicas relacionadas a atividade laboral, são ameaças constantes à saúde do trabalhador (Jacinto et al., 2020). A EDT apresenta uma lista de disfunções psicológicas e do corpo e avalia a opinião dos trabalhadores sobre dores dos membros superiores e inferiores, perda da autoconfiança, dificuldade nas decisões da vida, insônia, dentre outros, em decorrência de experiências negativas do trabalho (Facas, 2013). Os fatores de risco geralmente estão associados a aspectos que estão presentes diariamente na vida do trabalhador, tais como problemas de saúde, tanto físico quanto psicossociais, relacionados ao trabalho. Esses fatores são revelados de acordo com a complexidade do trabalho, as altas demandas e variações de atividades, podendo favorecer o surgimento de doenças (Cardoso \& Bakke, 2018). A EDT é avaliada da mesma forma que a escala que a EIST. A Tabela 5 destaca os resultados mais representativos da escala.

Tabela 5

Escala de Danos Físicos e psicossociais no trabalho (EDT)

\begin{tabular}{llll}
\hline Fator - Item & Média & DP & Risco \\
\hline Danos Psicológicos & $\mathbf{1 , 8 6}$ & $\mathbf{0 , 8 9}$ & Baixo \\
\hline Danos Sociais & $\mathbf{1 , 9 1}$ & $\mathbf{0 , 7 9}$ & Baixo \\
\hline Danos Físicos & $\mathbf{2 , 6 5}$ & $\mathbf{1 , 1 3}$ & Médio \\
\hline Alteração no apetite. & 2,43 & 1,57 & Médio \\
Dores nas costas. & 2,68 & 1,56 & Médio \\
Dor de cabeça. & 2,89 & 1,44 & Médio \\
Alterações no sono. & 2,89 & 1,55 & Médio \\
Dores no corpo. & 2,93 & 1,35 & Médio \\
\hline
\end{tabular}


Dores no braço.

Dores nas pernas.

$\mathrm{N}=28 ; \mathrm{DP}=$ Desvio Padrão

Os três fatores que compõem a escala são: danos psicológicos $(M=1,86 ; D P=0,89)$ que caracteriza sentimentos negativos sobre a vida em geral e sobre si; danos sociais $(M=$ $1,91 ; D P=0,79)$ em que os itens representam dificuldades nas relações sociais e comportamentos de isolamento; e danos físicos $(M=2,65 ; \quad D P=1,13)$ que apresenta afirmações sobre dores físicas e distúrbios biológicos (Facas \& Mendes, 2018; Silva, 2018). De acordo com Huebra et al. (2018), o conceito de saúde do trabalhador não é fixo e estanque, porém pode ser compreendido como resultado da saúde física, social e psicológica, onde a mente é responsável pelo corpo e ambos devem andar juntos.

Os fatores "danos psicológicos" e "danos sociais" não obtiveram itens avaliados como riscos psicossociais médio ou alto. Silva (2018) traz que danos psicológicos e sociais podem acarretar comportamentos reprováveis, assim o trabalhador pode ficar exposto com sua posição de trabalho ameaçada, sendo levado em consideração que os danos podem ser causados por diversas coisas, a principal é as lideranças autoritárias.

O fator "danos físicos" apresentou itens com risco médio, o que indica a necessidade de atenção e intervenção, estando relacionados a alteração no apetite, no sono, dores nas costas, cabeça, corpo, braço e pernas. Para esses aspectos pode ser feita uma avaliação ergonômica dos postos de trabalho da empresa, com programas simples de correção na postura, alongamentos e alimentação saudável (Battiston, Cruz, \& Hoffmann, 2006). Ações de promoção a saúde relacionadas ao reconhecimento, à prevenção e ao tratamento de doenças profissionais são constantemente recomendadas quando se identifica fatores de risco no ambiente de trabalho (Jacinto et al., 2020), ou seja, este reconhecimento e controle dos riscos fomenta a efetiva promoção do trabalho como fonte de emancipação e de dignidade humana (Zanelli \& Kanan, 2018).

\section{Considerações Finais}

Este estudo mapeou os riscos psicossociais no trabalho de auxiliares de cozinha por meio de um protocolo composto por quatro escalas que investigaram as características da organização do trabalho, o estilo de gestão, os indicadores de sofrimento e os danos físicos e psicossociais decorrentes do trabalho. A escala que avaliou a organização do trabalho, ou seja, que possuía itens relacionados as condições, prazos, ritmos, normas, responsabilidades e divisão do trabalho evidenciou riscos psicossociais mais elevados. Nessa escala, aspectos que descreveram sobre a quantidade trabalhadores para a execução das tarefas, ritmo de trabalho e a participação dos trabalhadores na tomada de decisão apresentaram-se como alto risco psicossocial.

A escala sobre a gestão predominante na organização, com itens que avaliavam o controle no trabalho, hierarquia e regras, evidenciou que as características dos dois estilos apresentados - individualista e coletivista - possuem presença moderada na empresa estudada. Apesar disso, os itens relacionados ao estilo de gestão coletivista foram assinalados como mais presentes, dessa forma é válido ressaltar que os gestores nessa organização valorizam o trabalho em grupo, reconhecem as conquistas como mérito de todos e levam em conta o bem-estar dos trabalhadores.

Os itens avaliados pelas escalas relacionadas aos indicadores de sofrimento e aos danos físicos e psicossociais no trabalho mostraram-se, de maneira geral, como baixos riscos. Porém deve ser destacado aspectos relacionados ao esgotamento mental, tais como o desânimo, a sobrecarrega, o desgaste e o 
cansaço que indicaram risco psicossocial médio, bem como deve-se levar em conta as dores físicas que as trabalhadoras relataram, podendo levar a riscos mais graves no futuro. Avaliar as condições de trabalho, principalmente ergonômicas, e realizar uma análise de cargos dos trabalhadores poderá melhorar a saúde e esclarecer as reais demandas de trabalho e das funções. O monitoramento dos riscos psicossociais no trabalho deve ser um processo contínuo na rotina das organizações, envolvendo todas as partes interessadas. A identificação produz e mobiliza conhecimentos sobre esses fatores e permite planejar e implementar medidas que visem impedir que o risco se transforme em adoecimento ao trabalhador.

Como limitações da pesquisa, acreditase que, contou-se com uma amostra pequena mesmo com quase todos os trabalhadores que atendiam aos critérios estabelecidos - o que restringiu a utilização de análises estatísticas elaboradas e comparações de médias entre grupos. Durante a aplicação do protocolo percebeu-se que algumas trabalhadoras ficaram desconfiadas, com receio que as respostas individuais fossem entregues a gestão da empresa, o que pode ter trazido um viés nos dados. Como desenvolvimento de pesquisas futuras, sugere-se comparar/relacionar respostas de cozinheiros - que atuam em cargos de gestão/liderança - e dos auxiliares, assim seria possível verificar se há diferenças nas percepções de riscos, entre esses públicos. Também podem ser desenvolvidas pesquisas que ampliem a amostra, comparando com outras empresas e estados do país, já que estudos com categorias profissionais que, muitas vezes, são invisibilizadas - com a de auxiliares de cozinha - são essenciais pois propiciam um reconhecimento da importância da função, bem como podem fornecer subsídios teóricos para que a gestão promova ações de melhoria das condições e da organização do trabalho a que são expostos esses profissionais

\section{Referências}

Álvarez, C. C. B. (2012). Riesgo psicossocial intralaboral y burnout en docentes universitarios de algunos países latinoamericanos. Cuadernos de Administración, 28(48), 117-132. Recuperado de http://www.scielo.org.co/pdf/cuadm/v28n4 8/v28n48a10.pdf

Amorim, T. G. F. N. (2010). Qualidade de vida no trabalho: Preocupação também para servidores públicos? Revista Eletrônica de Ciência Administrativa, 9(1), 35-48. doi: 10.5329/RECADM.20100901003

Battiston, M., Cruz, R. M., \& Hoffmann, M. H. (2006). Condições de trabalho e saúde de motoristas de transporte coletivo urbano. Estudos de Psicologia, 11(3), 333-343. doi: 10.1590/S1413-294X2006000300011

Cardoso, A. W. M., \& Bakke, H. A. (2018). Estresse ocupacional em profissionais de saúde dos centros de atenção psicossocial. Revista Brasileira de Saúde e Segurança no Trabalho, 1(1), 23-29. Recuperado de http://periodicos.ifpb.edu.br/index.php/rebr ast/article/view/1552

Carlotto, P. A. C., Cruz, R. M., Guilland, R., da Rocha, R. E. R., Dalagasperina, P., \& Ariño, D. O. (2018). Riscos Psicossociais Relacionados ao Trabalho: perspectivas teóricas e conceituais. Revista

Interamericana de Psicología Ocupacional, 37 (1), 52. doi: 10.21772/ripo.v37n1a04

Ceribeli, H. B., \& Rocha. G. B. de S. (2019). Uma análise da relação entre flexibilização do trabalho, exaustão emocional e engajamento dos trabalhadores. Revista Ciências Administrativas, 25(1), 1-14. doi: 10.5020/2318-0722.2019.7420

Conte, A. L. (2003). Qualidade de vida no trabalho: Funcionários com qualidade de vida no trabalho são mais felizes e produzem mais. Revista FAE Business, 7 , 32-34. Recuperado de https://www.docsity.com/pt/qualiidade-deviida-no-trabalho/4758527/ 
Duarte, R. F. (2018). Os riscos psicossociais no trabalho e as políticas públicas de preservação da saúde mental do trabalhador (Dissertação de mestrado, Universidade Estadual Paulista Júlio de Mesquita Filho). Recuperado de https://repositorio.unesp.br/handle/11449/1 80559

Facas, E. P. (2013). Protocolo de avaliação dos riscos psicossociais no trabalho: Contribuições da psicodinâmica do trabalho (Tese de doutorado, Universidade de Brasília). Recuperado de https://repositorio.unb.br/handle/10482/154 $\underline{20}$

Facas, E. P., \& Mendes, A. M. (2018).

Estrutura fatorial do protocolo de avaliação dos riscos psicossociais no trabalho. Núcleo Trabalho, Psicanálise e Crítica Social.

Recuperado de http://nucleotrabalho.com.br/wpcontent/uploads/2018/12/Facas-MendesEstrutura-Fatorial-do-Protocolo-deAvalia\%C3\%A7\%C3\%A3o-dos-RiscosPsicossociais-no-Trabalho1.pdf

Facas, E. P., Duarte, F. S., Mendes, A. M., \& Araujo, L. K. R. (2015). Sofrimento ético e (in) dignidade no trabalho bancário: Análise clínica e dos riscos psicossociais. In J. K. Monteiro, F. de O. Vieira, \& A. M. Mendes (Orgs.), Trabalho \& Prazer: Teoria, Pesquisas e Práticas (pp. 233-255). Curitiba: Juruá Editora.

Faria, J. H. de. (2017). Autogestão, economia solidária e organização coletivista de produção associada: Em direção ao rigor conceitual. Cadernos EBAPE.BR, 15(3), 629-650. doi: 10.1590/1679-395157778

Gomez, C. M., \& Costa, S. M. da F. T. (1997). A construção do campo da saúde do trabalhador: Percurso e dilemas. Cadernos de Saúde Pública, 13(2), 21-32. doi: 10.1590/S0102-311X1997000600003

Gomez, C. M., Vasconcellos, L. C. F. de, \& Machado, J. M. H. (2018). Saúde do trabalhador: Aspectos históricos, avanços e desafios no Sistema Único de Saúde. Ciência \& Saúde Coletiva, 23(6), 19631970. doi: $\underline{10.1590 / 1413-}$ $\underline{81232018236.04922018}$
Huebra, P. da M., Ribeiro, C. A., Crescencio, L. N. P., Sampaio, R. M. F., Silva, R. M. G. da C. S., Martins, W. S. O., ... Damasceno, M. R. (2018). Condições de saúde mental e física do trabalhador. IV Seminário Científico da FACIG. Recuperado de http://pensaracademico.facig.edu.br/index.p hp/semiariocientifico/article/view/782

Jacinto, A., Huck, C. K., Silva, M. G. da., \& Tolfo, S. R. (2020). Fenômenos psicossociais relacionados ao trabalho: promovendo saúde e monitorando riscos. In S. da R. Tolfo (Orgs), Gestão de Pessoas e Saúde Mental do trabalhador: fundamentos e intervenções com base na Psicologia (pp. 203- 221). São Paulo: Vetor Editora.

Lino, M. H. F. V. (2018). Satisfação com o trabalho dos profissionais de enfermagem na unidade local de saúde do Nordeste (Relatório de Estágio, Instituto Politécnico de Bragança). Recuperado de https://bibliotecadigital.ipb.pt/handle/10198 /19104

Mendes, M. de M. (2016). Condições de trabalho e riscos psicossociais: Um estudo com os funcionários do restaurante universitário da Universidade Federal do Rio Grande do Norte (Dissertação de mestrado, Universidade Federal do Rio Grande do Norte). Recuperado de https://repositorio.ufrn.br/jspui/handle/1234 56789/22185

Merlo, A. R. C., \& Mendes, A. M. B. (2009). Perspectivas do uso da psicodinâmica do trabalho no Brasil: Teoria, pesquisa e ação. Cadernos de Psicologia Social e do Trabalho, 12(2), 141-156. doi: 10.11606/issn.1981-0490.v12i2p141-156

Organização Internacional do Trabalho [OIT]. (2010). Health impact of psychosocial hazards at work: An overview. Institute of Work, Health \& Organisations, University of Nottingham. Recuperado de https://apps.who.int/iris/bitstream/handle/1 0665/44428/9789241500272_eng.pdf.

Pacheco, T. P., \& Silva, R. M. P. da. (2018). Risco psicossocial para servidores de universidade pública na região norte do Brasil. Revista Psicologia: Organizações e 
Trabalho, 18(1), 335-344. doi:

10.17652/rpot/2018.1.13388

Pucci, S. M., Kanan, L. A., \& Silva, B. F. da. (2017). Riscos psicossociais no contexto do trabalho. Revista Gepes Vida, 3(6), 142-

153. Recuperado de http://www.icepsc.com.br/ojs/index.php/ge pesvida/article/view/239

Rodrigues, L. B., \& Santana, N. B. (2010). Identificação de riscos ocupacionais em uma indústria de sorvetes. Unopar Científica, 12, 1-18. Recuperado de https://pgsskroton.com.br/seer/index.php/J HealthSci/article/viewFile/1294/1239

Ruiz, V, S., \& Araujo, A. L. L. de. (2012). Saúde e segurança e a subjetividade no trabalho: Os riscos psicossociais. Revista Brasil Saúde Ocupacional, 37(125), 170180. doi: $10.1590 / \mathrm{S} 0303$ 76572012000100020

Silva, A. P. B. (2018). Riscos e danos relacionados ao contexto do trabalho da equipe de enfermagem de unidades neonatais (Dissertação de mestrado,
Universidade Federal de Goiás).

Recuperado de

https://repositorio.bc.ufg.br/tede/handle/ted e/8897

Silva, J. S. C., \& Garcia, F. C. (2018). Prazer e sofrimento no trabalho: Estudo com mulheres terceirizadas que atuam nos serviços gerais em uma instituição federal de ensino superior. Revista Brasileira de Administração Científica, 9(2), 36-49. doi: 10.6008/CBPC2179-684X.2018.002.0004

Tolfo, S. R. (2019). Significados e sentidos do trabalho. In P. F. Bendassolli, \& J. BorgesAndrade (Orgs.), Dicionário de Psicologia do Trabalho e das Organizações (pp. 617626). São Paulo: Artesã.

Vieira, F. de O., Mendes, A. M., \& Merlo, A. R. C. (Orgs.). (2013). Dicionário crítico de gestão e psicodinâmica do trabalho. Curitiba: Juruá Editora

Zanelli, J. C., \& Kanan, L. A. (2018). Fatores de risco, proteção psicossocial e trabalho: organizações que emancipam ou que matam. Florianópolis: Uniplac Editora

\section{Dados sobre as autoras:}

- Fabieli Tais Rüedell: Psicóloga pela IMED e pós graduanda em gestão de RH com ênfase em treinamento e desenvolvimento pela faculdade Unyleya. Atualmente atua na área organizacional e clínica.

- Júlia Gonçalves: psicóloga pela Universidade Franciscana, Mestre em Psicologia pela Universidade Federal de Santa Maria (UFSM) e Doutora em Psicologia pela Universidade Federal de Santa Catarina (UFSC). Atualmente é docente do Programa de Pós-Graduação (PPGP) e da Graduação em Psicologia da IMED e Pesquisadora Voluntária do Núcleo de Estudos de Processos Psicossociais e de Saúde nas Organizações e no Trabalho (NEPPOT/UFSC).

A submissão de originais para este periódico implica na transferência, pelos autores, dos direitos de publicação impressa e digital. Os direitos autorais para os artigos publicados são do autor, com direitos do periódico sobre a primeira publicação. Os autores somente poderão utilizar os mesmos resultados em outras publicações indicando claramente este periódico como o meio da publicação original. Em virtude de sermos um periódico de acesso aberto, permite-se o uso gratuito dos artigos em aplicações educacionais e científicas desde que citada a fonte conforme a licença CC-BY da Creative Commons. 